\title{
Ten Years of Liquid-phase Exfoliation of Layered Crystals - A Bright Future ahead?
}

\author{
Claudia Backes*
}

\begin{abstract}
Layered materials are an abundant source of new nanostructures when thinned down to display fascinating layer number-dependent properties due to quantum confinement in two dimensions. About ten years ago, it was first demonstrated that layered crystals can be exfoliated down to monolayers in the liquid phase by a relatively simple and scalable methodology termed liquid-phase exfoliation that is widely applicable to many structures. By now, $>10$ classes of layered materials have been made accessible as two-dimensional nanosheets by this technique. In this article, advancements in exfoliation, size selection and characterisation are summarised. Remaining challenges, open questions and perspectives are also discussed.
\end{abstract}

Keywords: 2D materials · Layered crystals · Liquid exfoliation · Nanosheets · Size control

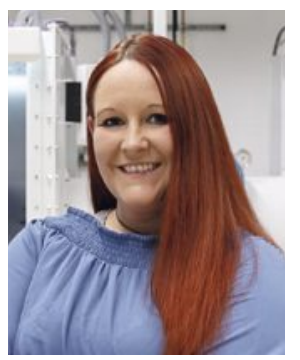

Claudia Backes obtained her $\mathrm{PhD}$ with honours in 2011 from the University of Erlangen, Germany. After some time as deputy executive director in the Erlangen Cluster of Excellence 'Engineering of Advanced Materials', Claudia received a fellowship grant from the German Research Foundation (DFG) in 2012 and moved to Jonathan Coleman's group at Trinity College Dublin, Ireland. In 2015, she returned to Germany and started her independent research at the Chair of Applied Physical Chemistry at Heidelberg University funded through the prestigious Emmy Noether grant by the German Research Foundation from 2016. Since 2011, her research has been devoted to liquid phase exfoliation of layered crystals, optical characterisation, deposition and functionalisation of nanosheets.

\section{Introduction}

In nature, not all crystals form chemical bonds in three dimensions. Many examples of so-called layered crystals are known, where strong chemical bonds are only formed in-plane resulting in platelets or sheets that are stacked into three-dimensional structures. Well-known examples are graphite, clays, or other minerals such as molybdenite. For centuries, humankind has been exploiting the properties of layered materials. Due to the weak out-ofplane bonding in the sheet stacks, layered crystals can be sheared parallel or expanded normal to the in-plane direction making them useful as dyes, lubricants or electrodes in batteries. In 2004 and 2005, Geim and Novoselov demonstrated that it is possible to isolate individual sheets from graphite that are only one atomic layer thick by micromechanical exfoliation. ${ }^{[1]}$ This marked the birth of a new research area that aims at isolating and characterising individual sheets, often referred to as $2 \mathrm{D}$ materials (even though strictly speaking, most of them are not one atomic layer thick). The exfoliation not only drastically increases the accessible surface area, but also constrains the electron wave function in two dimensions. This 2D quantum confinement dramatically changes the properties of the materials. One can even consider isolated

${ }^{*}$ Correspondence: Dr. C. Backes E-mail: backes@uni-heidelberg.de

Chair of Applied Physical Chemistry, Heidelberg University, Im Neuenheimer Feld 253, 69120 Heidelberg, Germany thin layers as new materials with properties that yet remain to be explored in many cases.

It is thus no surprise that tremendous research efforts have been devoted to synthesizing 2D materials by bottom up techniques or isolating them via top down approaches. One strategy that has become increasingly popular is exfoliation in liquids. ${ }^{[2]}$ The simplest form of this top down exfoliation is termed liquid-phase exfoliation (LPE). In this process, a layered crystal is immersed in a suitable solvent or aqueous surfactant solution and subjected to high-energy treatment such as sonication. The energy input overcomes the weak in-plane interactions between the sheets, while the solvent or surfactant adsorbs on the nanosheets thus preventing re-aggregation. No pre-treatment, intercalation or chemical modification of the bulk crystal is required making this a relatively simple and straight-forward process. In 2008, Coleman and co-workers demonstrated for the first time that graphite can be exfoliated down to monolayer graphene when the solvent is wisely chosen. ${ }^{[3]}$ According to solution thermodynamics, this is the case when the solubility parameters of solvent and solute match. By screening a range of organic solvents, a handful of candidates were identified (for example $N$-methyl-2-pyrrolidone) that efficiently prevent nanosheet aggregation. Shortly after, the same group reported that aqueous surfactant solutions can also be used. ${ }^{[4]}$ In this case, the hydrophobic part of the amphiphiles adsorbs on the nanosheet, while the polar head groups point in the aqueous medium resulting in stabilisation of the colloid. Importantly, the very same strategy can be applied to other layered crystals such as transition metal dichalcogenides or hexagonal boron nitride. ${ }^{[5]}$ While this was not the first time that colloidally stable dispersions of transition metal dichalcogenides were characterised, ${ }^{[6]}$ it is widely considered as the birth of liquid-phase exfoliation.

\section{Why Liquid-phase Exfoliation}

A great advantage of liquid-phase exfoliation is that, in its crudest form, it is a relatively simple protocol that does not require expensive equipment or extraordinary experimental skills making it accessible to researchers worldwide. The inherent solution processability can be exploited to fabricate composites by simply mixing dispersions with different constituents. This is probably one of the greatest strengths of LPE, as it allows to explore synergistic effects that arise when combining materials with various properties and dimensionality. Of equal or maybe even greater importance is the versatility. LPE can not only be applied to layered crystals that are widely studied in their 2D form (e.g. graph- 
ite, hexagonal boron nitride, group VI transition metal dichalcogenides such as $\mathrm{MoS}_{2}$, black phosphorus), but also to more exotic structures (see Fig. 1). Examples are layered hydroxides (e.g. $\mathrm{Ni}(\mathrm{OH})_{2}{ }^{[7]}$ or $\left.\mathrm{Co}(\mathrm{OH})_{2}{ }^{[8]}\right)$, other transition metal dichalcogenides (e.g. $\left.\mathrm{TiS}_{2},{ }^{[9]} \mathrm{TaS}_{2},{ }^{[10]} \mathrm{ReS}_{2}{ }^{[11]}\right)$, III-VI (e.g. GaS, ${ }^{[12]} \mathrm{InSe}^{[13]}$ ) or IVVI $\left(\mathrm{SnS},{ }^{[14]} \mathrm{GeTe}^{[15]}\right)$ semiconductors, layered oxides $\left(\mathrm{MoO}_{2},{ }^{[16]}\right.$ $\left.\mathrm{MoO}_{3}{ }^{[17]}\right)$, transition metal phosphorus trisulfides (e.g. $\left.\mathrm{NiPS}_{3}{ }^{2}{ }^{[18]}\right)$ or layered silicates ${ }^{[19]}$ (such as talc or mica). Importantly, LPE does not require expensive, often not accessible high quality large area crystals. Powders or even impure, naturally occurring starting materials can be used, as only the layered portion of a crystal will be exfoliated and stabilised.[19] As such, the properties of 2D nanosheets can be explored that are not easily accessible by other production techniques.

From LPE dispersions, inks can be formulated and deposited by various techniques such as spray coating, inkjet, screen or gravure printing. ${ }^{[20]}$ This has resulted in the demonstration of proof of concept applications of LPE nanosheets from a range of layered crystals in diverse areas from nanocomposites, (opto)electronics and photonics to sensing and energy storage and conversion. For a detailed description, the reader is referred to recent review articles. ${ }^{[20,21]}$ The interest in exploring the application potential of the never-ending supply of new nanostructures has called for an increasing demand in producing gram quantities of the nanosheets. On the laboratory scale, sonication is widely used to supply the energy to overcome the interaction between the layers. However, even high-power tip sonication typically only yields milligram quantities. Therefore, large-scale production techniques are being developed or adapted from industry. ${ }^{[22]}$ Examples are shear exfoliation in rotor stator mixers ${ }^{[23]}$ or by compressive flow, ${ }^{[24]}$ ball milling, ${ }^{[25]}$ or microfluidisation ${ }^{[26]}$ to name just a few.

\section{Size and Thickness Control: The Main Bottleneck}

The major drawback of nanosheet dispersions from LPE is that size and thickness distributions are very broad with lateral dimensions from few nanometres to micrometres and 1-30 layers or more. Since the size, and in particular thickness, determines the properties of a material, this is a severe issue. Therefore, post exfoliation size selection for example by centrifugation is required. In early works, the dispersions were typically centrifuged in only one step at relatively low centrifugal acceleration in a homogeneous centrifugation and the supernatant decanted and collected (Fig. 2A). ${ }^{[3-5]}$ While this removes unexfoliated material in the sediment, the size and thickness control is poor. If the dispersion is centrifuged at low centrifugal acceleration, both large and small nanosheets will not sediment and be found in the supernatant. Smaller/thinner nanosheets can be isolated by centrifuging at higher centrifugal acceleration, but then the majority of the material is wasted and discarded. A significant improvement over this strategy was proposed a few years ago. The idea is to perform centrifugation in a cascade with iteratively increasing centrifugal acceleration (liquid cascade centrifugation, LCC, Fig. 2B). [27] After each step, supernatant and sediment are separated, the sediment collected in fresh solvent/surfactant and the supernatant subjected to centrifugation at higher centrifugal acceleration. In this way, small nanosheets are also removed from dispersions centrifuged at low centrifugal acceleration to isolate larger nanosheets with narrower distribution. The great advantage is that a range of sizes/thicknesses can be isolated from one initial (stock) dispersion without wastage of material. In addition, redispersion of the sediment has the advantage that the concentration of the nanosheets can be adjusted and the medium exchanged.
Graphite
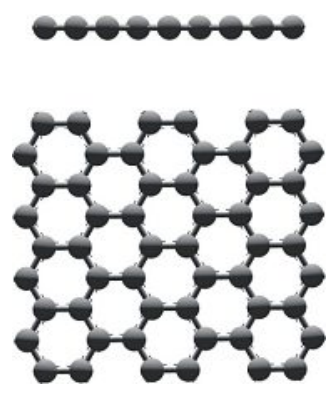

III-VI

semiconductors
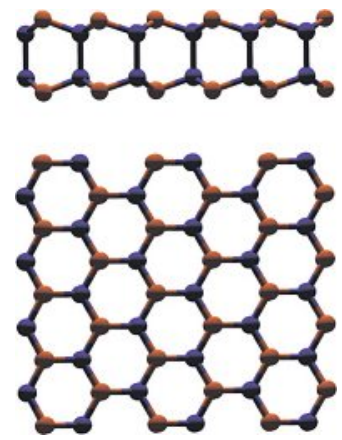

h-BN

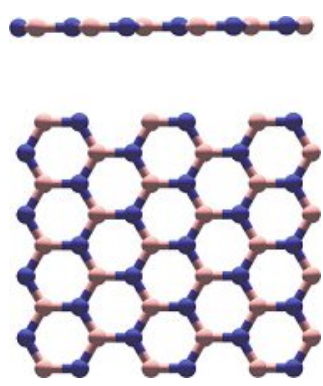

IV-VI

semiconductors
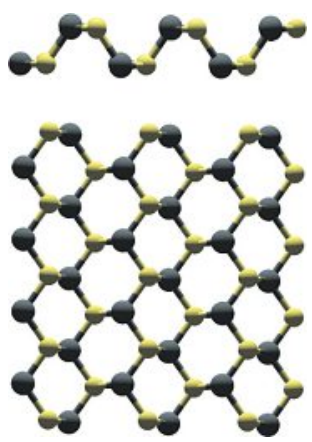

Pnictogens

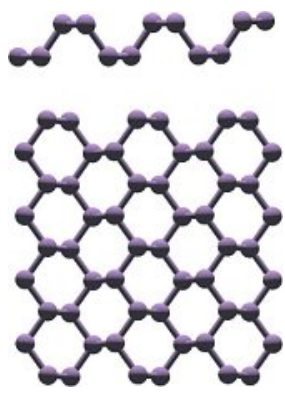

Oxides $\left(\mathrm{MoO}_{3}\right)$
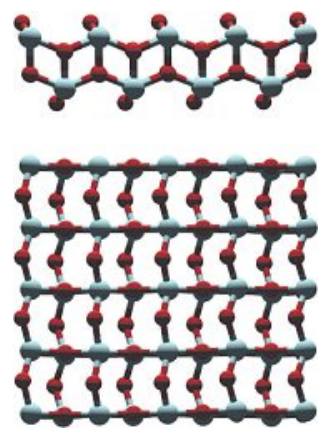

$2 \mathrm{H}-\mathrm{TMDs}$
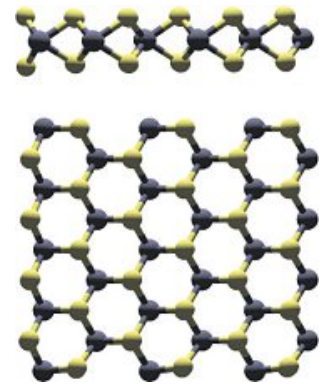

Transition metal phosphorus trisulfides
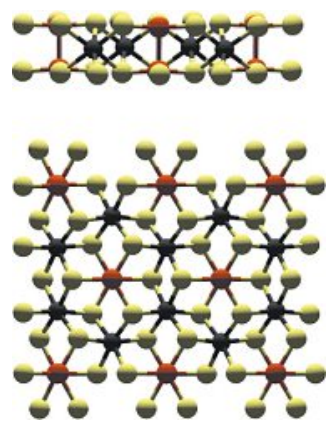

1T-TMDs
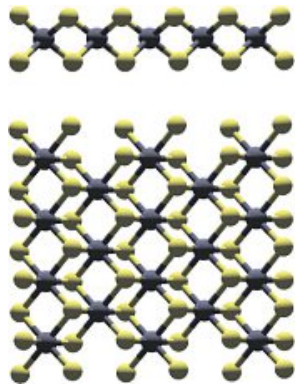

Hydroxides

$\left(\mathrm{Co}(\mathrm{OH})_{2}\right)$

Fig. 1. Examples of structures of layered materials that were exfoliated by liquid exfoliation in side and top view. Graphite; Hexagonal boron nitride (blue: nitrogen, red: boron); Pnictogens (e.g. black phosphorus or antimony); Transition metal dichalcogenides (TMDs; black: transition metal, yellow: chalcogen) crystallising preferentially as $2 \mathrm{H}\left(\mathrm{MoS}_{2}, \mathrm{WS}_{2}, \mathrm{MoSe}_{2}, \mathrm{WSe}_{2}\right)$ or $1 \mathrm{~T}$ polytype $\left(\mathrm{TiS}_{2}, \mathrm{TaS}_{2}, \mathrm{ReS}\right)$; III-VI semiconductors (InSe or GaS; blue: metal, red: chalcogen); IV-VI semiconductors (SnS, GeTe; yellow: chalcogen; dark green: Sn); transition metal trioxides (MoO; ${ }_{3}$ cyan: Mo, red: oxygen); transition metal phosphorus trisulfides (NiPS 3 ; black: transition metal, orange: phosphorus, yellow: chalcogen); hydroxides (Co(OH); red: oxygen, grey: hydrogen). 

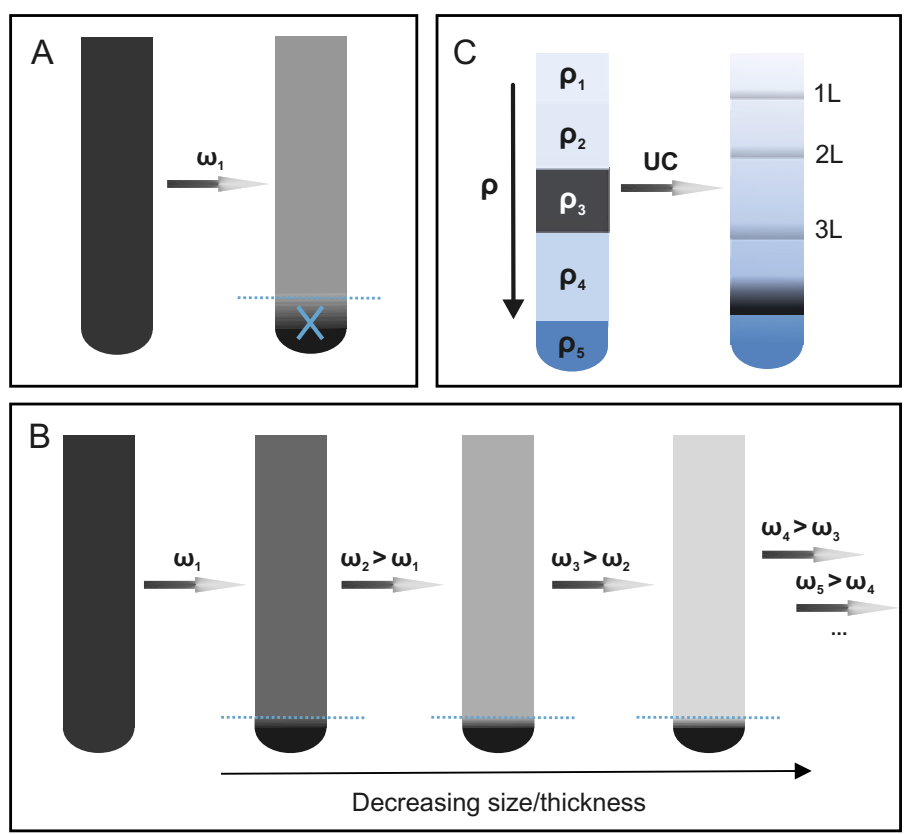

Fig. 2. Schematic representation of size-selection schemes for liquidexfoliated nanosheets. A) Homogeneous centrifugation, B) liquid cascade centrifugation, C) density gradient ultracentrifugation.

Since only minor modifications in the centrifugation protocol are required for different materials or liquid media, liquid cascade centrifugation is now widely used. However, a problem remains: overall, the constituents in the dispersion are size-selected by their hydrodynamic volume. This means that small and thin nanosheets are separated from larger/thicker ones. While modifications of the procedure in secondary cascades show some potential to isolate larger and yet thin nanosheets, ${ }^{[27]}$ LCC does not allow for a separation strictly by layer number. This would require a size selection by buoyant density rather than hydrodynamic volume. This is because the buoyant density includes contributions from the solvent/surfactant shell, which will therefore be sensitive to the layer number. Buoyant density separation can be achieved by density gradient ultracentrifugation (DGU, Fig. 2C). The proof of concept was first demonstrated by Hersam and co-workers in 2009 in the case of graphene. [28] In DGU, layers of decreasing density from bottom to top are pre-formed in the centrifuge tube by the addition of a density gradient medium (typically iodixanol or other sugars) of various concentration. On ultracentrifugation $(>200,000 \mathrm{~g})$, a density gradient is formed in the vial. When centrifuging to equilibrium (overnight), the nanomaterial previously inserted into the pre-formed step gradient moved to the position in the centrifuge tube where the buoyant density of the material matches the density of the gradient (isopycnic centrifugation). In the case of $2 \mathrm{D}$ materials, this ideally results in the formation of bands that are well separated from each other. Monolayers have the lowest buoyant density and will be accumulated in the top band, followed by bilayers, trilayers, etc. For the separation to be successful, the density gradient in the medium has to be carefully tuned to the densities of the materials to be separated. High density materials such as $\mathrm{MoS}_{2}$ lie outside the density window that is accessible by density gradient media when exfoliated and stabilised in typical monomeric surfactants. ${ }^{[29]}$ In this case, a disordered polymer coating on the nanosheets is required to lower the buoyant density of the nanosheets. Even though DGU is thus far the only method that can sort nanosheets in dispersion by layer number, it is not widely used. This is probably because the process is delicate, yields are low and density gradient and/or polymer coating are difficult to remove.

\section{Characterising an Ensemble: Strength and Weakness}

No matter how nanosheets are size-selected, the size and thickness distribution will never be monomodal and some polydispersity in dimensions will remain. This means that only properties of an ensemble are accessible. In spectroscopic characterisation, this can be an advantage as millions of nanosheets are probed in a single measurement and variation across nanosheets, for example due to inhomogeneous environment or defects, ${ }^{[30]}$ are averaged out. However, the polydispersity also implies that a precise statistical measurement of the nanosheet dimensions is required when insights into size- and thickness-dependent properties are targeted.

The accurate size and thickness measurement is still a challenge that many researchers in the field face. Due to the non-uniform aspect ratios, techniques such as dynamic light scattering only find limited use, as they cannot distinguish between nanosheet lateral size and layer number. Therefore, microscopic techniques are the tool of choice. Large area micromechanically-cleaved or CVD grown 2D materials can often be characterised by white light microscopy on opaque bi-layered substrates such as $\mathrm{Si} / \mathrm{SiO}_{2}$ due to light interference. ${ }^{[31]}$ However, LPE nanosheets are laterally too small to be clearly resolved. Instead, due to the large field of view and nonetheless excellent resolution, transmission electron microscopy (TEM) is widely used to visualise the outcome of LPE and size selection. Since TEM can be combined with further analysis (such as diffraction, electron energy loss spectroscopy, etc.), it is a powerful characterisation technique. However, it fails to accurately determine the layer number. Initially, TEM was used to estimate layer numbers by analysing steps at flake edges. ${ }^{[3]}$ Due to the non-uniform thickness across the nanosheets, which tend to be thinner towards the edges, where the analysis is performed, this is associated with a large uncertainty.

For a nanosheet thickness analysis, atomic force microscopy (AFM) therefore appears a natural and intuitive choice. A few obstacles had to be overcome for this technique to become reliable. Firstly, nanosheet aggregation has to be prevented on deposition. This can be achieved by flash-evaporating the solvent as visualised in a video publication. ${ }^{[32]}$ Secondly, due to the limited field of view in scanning probe techniques, it is important to have means of pre-selecting promising areas for AFM. While optical micrographs do not have sufficient resolution for a precise analysis, deposition on opaque bilayered substrates is beneficial and can act as a useful guide. ${ }^{[33]}$ Thanks to van der Waals interactions, nanosheets adhere strongly to the substrate. As a result, they tend to lie down and washing steps can be implemented to remove solvent/surfactant impurities as illustrated in Fig. 3. Fig. 3A shows a typical wide view AFM image of a LPE graphite stock dispersion exfoliated in water surfactant by tip sonication prior to size selection. The polydispersity in nanosheet size, thickness and shape is clearly visible. Fig. 3B and 3C display AFM images of the same sample after liquid cascade centrifugation with larger/thicker nanosheets isolated at low centrifugal acceleration (Fig. 3B) and small/thin nanosheets isolated at high centrifugal acceleration (Fig. 3C). Size and thickness distributions are visibly narrowed.

As described above, LCC not only allows for a size selection, but the redispersion of the sediment has further advantages. For example, nanosheets can be redispersed at any desired concentration. Equally importantly, the medium can be exchanged or modified. For surfactant-based dispersions, this means that the surfactant concentration can be minimised. In the case of solvent-based dispersion, the solvent can be exchanged. One of the most popular and efficient solvents for LPE is $N$-methyl-2pyrrolidone (NMP) which has a high boiling point $\left(>200{ }^{\circ} \mathrm{C}\right)$ and can undergo sonopolymerisation. As a result, when nanosheets are directly deposited from NMP, aggregates are often problematic and even more critical, they tend to be covered in impurities 

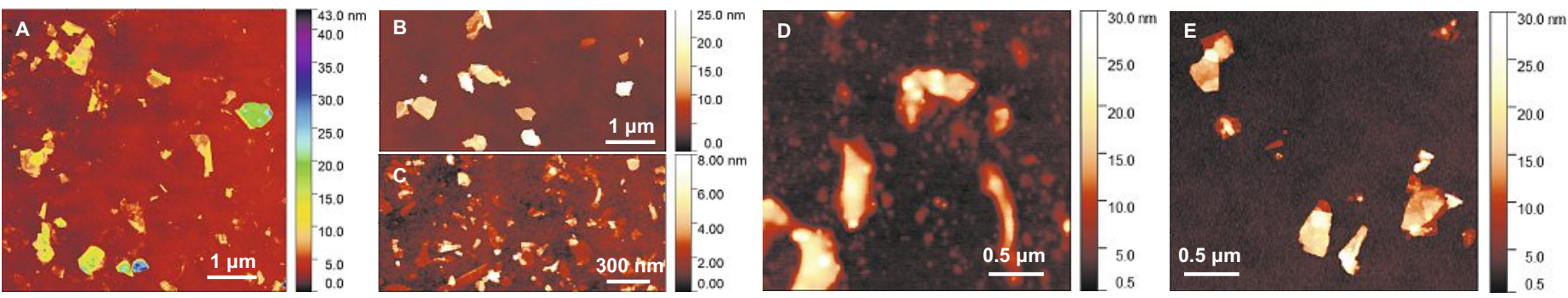

Fig. 3. Atomic force microscopy of liquid-exfoliated nanosheets. A) Wide view image of a graphite dispersion exfoliated in aqueous surfactant prior to size selection. B,C) Same sample after size selection by liquid cascade centrifugation to isolate large/thick nanosheets at low centrifugal acceleration (B) and small/thin nanosheets at high centrifugal acceleration (C). D) AFM image of graphite directly deposited from the solvent NMP showing solvent residues that do not allow for a size/thickness determination. E) Same samples as in D redispersed in isopropanol after size selection resulting in significantly improved image quality.

that cannot be removed by washing the wafer unlike surfactants. A typical AFM image of graphite exfoliated and deposited from NMP is shown in Fig. 3D. Clearly, no accurate analysis of nanosheet size and thickness is possible. However, when the sediment after LCC is collected in isopropanol and then deposited (Fig. $3 \mathrm{E})$, the wafer surface is widely free of contaminants and nanosheets with well-defined shapes and sharp edges can be imaged. Such good quality images can then be analysed and nanosheet length (longest dimension), width (dimension perpendicular) and thickness measured either manually ${ }^{[32]}$ or supported by image analysis software ${ }^{[34]}$ for a statistical evaluation of the dimensions of the nanosheets in the dispersion. To convert the apparent, measured AFM thickness to actual layer numbers, it needs to be considered that the AFM thickness is larger than the theoretical thickness due to potentially intercalated solvent, as well as capillary forces and adhesion. An analysis of steps associated with terraces of incompletely exfoliated nanosheets can be carried out for a calibration. [33]

Once lateral size and layer number distributions are known, it is possible to quantitatively study fundamental size and thickness dependent properties, for example by optical spectroscopy. Here, probing the ensemble can be an advantage. 2D materials will be very sensitive to any structural defects and to the dielectric environment. This complicates the investigation of intrinsic properties. When individual nanosheets obtained by micromechanical cleavage or growth are studied, a large variation in optical and electronic properties for materials with nominally identical layer numbers and chemical composition is observed. In ensemble measurements on LPE samples, these variations are averaged out. The most readily accessible characterisation technique for LPE nanosheets is extinction/absorbance spectroscopy. From a few show-cases, it is clear that the wavelength-dependent steady-state optical response of the nanosheets is influenced by both nanosheet lateral size and layer number. For example, edge effects result in changes in absorbance/extinction coefficients as function of wavelength. ${ }^{[9 b, 12,27,35]}$ As a result, once calibrated by statistical lateral size assessment, quantitative metrics can be derived to determine the mean lateral size of nanosheets by peak intensity ratios. In addition, nanosheet thickness was shown to impact the exciton energy due to confinement and dielectric screening effects. ${ }^{[12,27,35]}$ While such effects were observed also for micromechanically-exfoliated material, ${ }^{[36]}$ LPE nanosheets have the potential to shine further light on the layer number-dependent excitonic response due to the availability of a broad range of otherwise hardly accessible materials.

\section{Quo vadis Liquid-phase Exfoliation?}

In spite of the progress made in LPE over the past decade, it is not yet clear whether this process will result in a true scientific and technological advance. While LPE seems simple in its essence, care must be taken with post-exfoliation size selection and characterisation. Since a standardised procedure is currently lacking, it remains difficult to compare the outcome of the various exfoliation techniques that are proposed. A robust benchmarking will be required to unravel strengths and weaknesses of various approaches. To this end, the spectroscopic size and thickness metrics are potentially a way forward. Furthermore, it is well accepted that the reduced dimensionality will make the properties of $2 \mathrm{D}$ materials extremely sensitive to defects on the atomic scale as well as imperfections on the nano- or microscale. While this is an intrinsic issue with $2 \mathrm{D}$ materials, ${ }^{[30]}$ the question arises whether we can expect 'intrinsic' nanosheet properties in LPE samples, where sonication(exfoliation)-induced scission, i.e. tearing of the sheets in plane, likely occurs to some extent. The reasonably narrow room temperature photoluminescence linewidth in combination with purely excitonic emission in LPE WS ${ }_{2},{ }^{[27]}$ seems to suggest that at least optical properties are widely pristine and nanosheets are of good quality, but further investigations on the broad palette of LPE nanosheets will be required.

A major concern for LPE probably is that it fails to provide laterally large and yet thin nanosheets. It should be noted that many applications (catalysis, energy storage, etc.) will not require laterally large monolayers. Nonetheless, it is fundamentally interesting to find out where the bottleneck is. Is it the size selection that is not efficient enough to select the minority population of large and thin sheets? Is there an intrinsic limitation from the exfoliation process that depends on the in-plane and out-of-plane binding strength? Is there an intrinsic lateral size limit of monolayers in the liquid at which sheets wrinkle, fold and eventually collapse? In which way would this be dependent on the chemical composition and/or surface functionalisation? With robust size selection and measurements in place, we can now begin to address these very fundamental questions in the future.

Making and isolating promising nanosheet constituents in dispersion is one thing. Depositing them is yet another. Most of the nanosheet networks deposited and characterised up to now consist of randomly restacked nanosheets in porous arrays. While these can be useful in certain application areas, this can clearly not be the end of the story. Why would you spend years of optimising exfoliation and size selection if you then randomly restack the constituents again? Clearly, we need additional deposition techniques, where the aim is to prevent the nanosheets from restacking. Either by placing them accurately beside each other or by embedding them in a matrix of a flexible, bendable material.

Even though or maybe because we have these challenges ahead, there is space to dream. We can think of constructing wondermaterials on demand. You may ask: Wondermaterial for which purpose? Answer: Any application you can think of! Imagine we extend our understanding of the properties and application potential of the hundreds of layered materials that were predicted to be exfoliable ${ }^{[37]}$ and by means of material comparisons, we can rationally predict how these properties change as function of layer number. This would give us a unique toolbox of Lego pieces of all colours and shapes. Imagine further that we learn 
to assemble them into new structures to create hierarchical super-structure with even different properties arising from synergistic effects. This would indeed allow us to create new wondermaterials by mixing and matching of various constituents. Surely, even in the best-case scenario of our dreams, we will not be able to come to perfection. When using inorganic layered materials that are synthesized under harsh reaction conditions, we may never have the perfection we would like to dream of. After all, when bulk materials are structurally imperfect, any nanosheet produced from them will also be. But who says we are restricted to layered inorganic crystals? Giving the wealth of structures of materials with different chemical composition that have been exfoliated by LPE, it can be anticipated that similar exfoliation, size selection and characterisation techniques can be applied to the whole host of organic sheet stacks such as 2D polymers or layered covalent organic frameworks that can be made at ever growing structural perfection by molecular design. ${ }^{[38]}$ Overall, whether or not the future of liquid exfoliation of layered crystals is bright remains yet to be seen, but exciting insights are definitely expected.

\section{Acknowledgements}

Support from the German research foundation (DFG) under grant agreement Emmy-Noether, BA4856/2-1 is acknowledged. C.B. thanks Kevin Synnatschke for help with figure preparation.

Received: May 16, 2019

[1] a) K. S. Novoselov, A. K. Geim, S. V. Morozov, D. Jiang, Y. Zhang, S. V. Dubonos, I. V. Grigorieva, A. A. Firsov, Science 2004, 306, 666; b) K. S. Novoselov, D. Jiang, F. Schedin, T. J. Booth, V. V. Khotkevich, S. V. Morozov, A. K. Geim, Proc. Natl. Acad. Sci. U. S. A. 2005, 102, 10451.

[2] V. Nicolosi, M. Chhowalla, M. G. Kanatzidis, M. S. Strano, J. N. Coleman, Science 2013, 340, 1420.

[3] Y. Hernandez, V. Nicolosi, M. Lotya, F. M. Blighe, Z. Sun, S. De, I. T McGovern, B. Holland, M. Byrne, Y. K. Gun'Ko, J. J. Boland, P. Niraj, G. Duesberg, S. Krishnamurthy, R. Goodhue, J. Hutchison, V. Scardaci, A. C. Ferrari, J. N. Coleman, Nat. Nanotechnol. 2008, 3, 563.

[4] M. Lotya, Y. Hernandez, P. J. King, R. J. Smith, V. Nicolosi, L. S. Karlsson, F. M. Blighe, S. De, Z. Wang, I. T. McGovern, G. S. Duesberg, J. N. Coleman, J. Am. Chem. Soc. 2009, 131, 3611.

[5] a) J. N. Coleman, M. Lotya, A. O’Neill, S. D. Bergin, P. J. King, U. Khan, K. Young, A. Gaucher, S. De, R. J. Smith, I. V. Shvets, S. K. Arora, G. Stanton, H.-Y. Kim, K. Lee, G. T. Kim, G. S. Duesberg, T. Hallam, J. J. Boland, J. J. Wang, J. F. Donegan, J. C. Grunlan, G. Moriarty, A. Shmeliov, R. J. Nicholls, J. M. Perkins, E. M. Grieveson, K. Theuwissen, D. W. McComb, P. D. Nellist, V. Nicolosi, Science 2011, 331, 568-571; b) R. J. Smith, P. J. King, M. Lotya, C. Wirtz, U. Khan, S. De, A. O’Neill, G. S. Duesberg, J. C. Grunlan, G. Moriarty, J. Chen, J. Wang, A. I. Minett, V. Nicolosi, J. N. Coleman, Adv. Mater. 2011, 23, 3944.

[6] M. Gutiérrez, A. Henglein, Ultrasonics 1989, 27, 259.

[7] A. Harvey, X. He, I. J. Godwin, C. Backes, D. McAteer, N. C. Berner, N. McEvoy, A. Ferguson, A. Shmeliov, M. E. G. Lyons, V. Nicolosi, G. S. Duesberg, J. F. Donegan, J. N. Coleman, J. Mater. Chem. C 2016, 4, 11046.

[8] D. McAteer, I. J. Godwin, Z. Ling, A. Harvey, L. He, C. S. Boland, V. VegaMayoral, B. Szydłowska, A. A. Rovetta, C. Backes, J. B. Boland, X. Chen, M. E. G. Lyons, J. N. Coleman, Adv. En. Mater. 2018, 1702965.

[9] a) X. Zhu, S. Chen, M. Zhang, L. Chen, Q. Wu, J. Zhao, Q. Jiang, Z. Zheng, H. Zhang, Photon. Res. 2018, 6, C44; b) V. Vega-Mayoral, R. Tian, A. G. Kelly, A. Griffin, A. Harvey, M. Borrelli, K. Nisi, C. Backes, J. N. Coleman, Nanoscale 2019, 11, 6206.

[10] Y. Liu, X. Ji, J. Liu, W. W. L. Tong, D. Askhatova, J. Shi, Adv. Funct. Mater. 2017, 27, 1703261.

[11] J. Kang, V. K. Sangwan, J. D. Wood, X. Liu, I. Balla, D. Lam, M. C. Hersam, Nano Lett. 2016, 16, 7216

[12] A. Harvey, C. Backes, Z. Gholamvand, D. Hanlon, D. McAteer, H. C. Nerl, E. McGuire, A. Seral-Ascaso, Q. M. Ramasse, N. McEvoy, S. Winters, N. C. Berner, D. McCloskey, J. Donegan, G. Duesberg, V. Nicolosi, J. N. Coleman, Chem. Mater. 2015, 27, 3483.
[13] E. Petroni, E. Lago, S. Bellani, D. W. Boukhvalov, A. Politano, B. Gürbulak, S. Duman, M. Prato, S. Gentiluomo, R. Oropesa-Nuñez, J.-K. Panda, P. S. Toth, A. E. Del Rio Castillo, V. Pellegrini, F. Bonaccorso, Small 2018, 14, 1800749.

[14] J. R. Brent, D. J. Lewis, T. Lorenz, E. A. Lewis, N. Savjani, S. J. Haigh, G. Seifert, B. Derby, P. O'Brien, J. Am. Chem. Soc. 2015, 137, 12689.

[15] P. Zhang, F. Zhao, P. Long, Y. Wang, Y. Yue, X. Liu, Y. Feng, R. Li, W. Hu, Y. Li, W. Feng, Nanoscale 2018, 10, 15989.

[16] J. B. Boland, A. Harvey, R. Tian, D. Hanlon, V. Vega-Mayoral, B. M. Szydłowska, A. Griffin, T. Stimpel-Lindner, S. Metel, V. Nicolosi, G. Duesberg, J. N. Coleman, Nanoscale Adv. 2019, 1, 1560.

[17] D. Hanlon, C. Backes, T. M. Higgins, M. Hughes, A. O’Neill, P. King, N McEvoy, G. S. Duesberg, B. Mendoza Sanchez, H. Pettersson, V. Nicolosi, J. N. Coleman, Chem. Mater. 2014, 26, 1751.

[18] R. Dangol, Z. Dai, A. Chaturvedi, Y. Zheng, Y. Zhang, K. N. Dinh, B. Li, Y Zong, Q. Yan, Nanoscale 2018, 10, 4890.

[19] A. Harvey, J. B. Boland, I. Godwin, A. G. Kelly, B. M. Szydłowska, G. Murtaza, A. Thomas, D. J. Lewis, P. O'Brien, J. N. Coleman, 2D Mater. 2017, 4, 025054

[20] a) G. Hu, J. Kang, L. W. T. Ng, X. Zhu, R. C. T. Howe, C. G. Jones, M. C. Hersam, T. Hasan, Chem. Soc. Rev. 2018, 47, 3265; b) F. Bonaccorso, A. Bartolotta, J. N. Coleman, C. Backes, Adv. Mater. 2016, $28,6136$.

[21] a) F. Bonaccorso, L. Colombo, G. Yu, M. Stoller, V. Tozzini, A. C. Ferrari, R. S. Ruoff, V. Pellegrini, Science 2015, 347, 1246501; b) V. B. Mohan, K.-t. Lau, D. Hui, D. Bhattacharyya, Composites Part B: Engin. 2018, 142, 200.

[22] M. Yi, Z. Shen, J. Mater. Chem. C 2015, 3, 11700.

[23] a) K. R. Paton, E. Varrla, C. Backes, R. J. Smith, U. Khan, A. O’Neill, C. Boland, M. Lotya, O. M. Istrate, P. King, T. Higgins, S. Barwich, P. May, P. Puczkarski, I. Ahmed, M. Moebius, H. Pettersson, E. Long, J. Coelho, S E. O’Brien, E. K. McGuire, B. M. Sanchez, G. S. Duesberg, N. McEvoy, T. J. Pennycook, C. Downing, A. Crossley, V. Nicolosi, J. N. Coleman, Nat. Mater. 2014, 13, 624; b) S. Biccai, S. Barwich, D. Boland, A. Harvey, D. Hanlon, N. McEvoy, J. N. Coleman, 2D Mater. 2018, 6, 015008.

[24] R. Rizvi, E. P. Nguyen, M. D. Kowal, W. H. Mak, S. Rasel, M. A. Islam, A. Abdelaal, A. S. Joshi, S. Zekriardehani, M. R. Coleman, R. B. Kaner, Adv Mater. 2018, 30, 1800200.

[25] a) C. Knieke, A. Berger, M. Voigt, R. N. K. Taylor, J. Röhrl, W. Peukert, Carbon 2010, 48, 3196; b) C. Damm, T. J. Nacken, W. Peukert, Carbon 2015, $81,284$.

[26] a) P. G. Karagiannidis, S. A. Hodge, L. Lombardi, F. Tomarchio, N. Decorde, S. Milana, I. Goykhman, Y. Su, S. V. Mesite, D. N. Johnstone, R. K. Leary, P. A. Midgley, N. M. Pugno, F. Torrisi, A. C. Ferrari, ACS Nano 2017, 11 2742; b) R. P. Keith, A. James, J. P. Andrew, S. Toby, Mater. Res. Expr. 2017, 4,025604 .

[27] C. Backes, B. M. Szydłowska, A. Harvey, S. Yuan, V. Vega-Mayoral, B. R. Davies, P.-1. Zhao, D. Hanlon, E. J. G. Santos, M. I. Katsnelson, W. J. Blau, C. Gadermaier, J. N. Coleman, ACS Nano 2016, 10, 1589.

[28] A. A. Green, M. C. Hersam, Nano Lett. 2009, 9, 4031.

[29] J. Kang, J.-W. T. Seo, D. Alducin, A. Ponce, M. J. Yacaman, M. C. Hersam, Nat. Commun. 2014, 5, 5478 .

[30] L. Zhong, R. C. Bruno, K. Ethan, L. Ruitao, R. Rahul, T. Humberto, A. P. Marcos, T. Mauricio, 2D Mater. 2016, 3, 022002.

[31] H. Li, J. Wu, X. Huang, G. Lu, J. Yang, X. Lu, Q. Xiong, H. Zhang, ACS Nano 2013, 7, 10344.

[32] C. Backes, D. Hanlon, B. M. Szydłowska, A. Harvey, R. J. Smith, T. M. Higgins, J. N. Coleman, J. Vis. Exp. 2016, 118

[33] C. Backes, T. M. Higgins, A. Kelly, C. Boland, A. Harvey, D. Hanlon, J. N. Coleman, Chem. Mater. 2017, 29, 243.

[34] L. Andrea, K.-A. Konstantinos, B. Xavier Diez, K. Alessandro, T. Emanuele, P. Nicola Maria, L. Giovanna De, G. Loris, P. Vincenzo, 2D Mater. 2017, 4, 025017.

[35] C. Backes, R. J. Smith, N. McEvoy, N. C. Berner, D. McCloskey, H. C. Nerl, A. O'Neill, P. J. King, T. Higgins, D. Hanlon, N. Scheuschner, J. Maultzsch, L. Houben, G. S. Duesberg, J. F. Donegan, V. Nicolosi, J. N. Coleman, Nat. Commun. 2014, 5, 4576.

[36] Y. Niu, S. Gonzalez-Abad, R. Frisenda, P. Marauhn, M. Drüppel, P. Gant, R. Schmidt, N. Taghavi, D. Barcons, A. Molina-Mendoza, S. de Vasconcellos, R. Bratschitsch, D. Perez De Lara, M. Rohlfing, A. Castellanos-Gomez, Nanomater. 2018, 8, 725

[37] N. Mounet, M. Gibertini, P. Schwaller, D. Campi, A. Merkys, A. Marrazzo, T. Sohier, I. E. Castelli, A. Cepellotti, G. Pizzi, N. Marzari, Nat. Nanotechnol. 2018, 13, 246 .

[38] a) X. Feng, A. D. Schlüter, Angew. Chem. Int. Ed. 2018, 57, 13748; b) W. Wang, A. D. Schlüter, Macromol. Rapid Commun. 2019, 40, 1800719. 